\title{
RANITIDINE THERAPY INCREASES THE RISK OF SEPSIS AND NECROTIZING ENTEROCOLITIS IN VERY LOW BIRTHWEIGHT INFANTS: PROSPECTIVE MULTICENTER STUDY
}

\author{
G. Terrin ${ }^{1}$, A. Passariello ${ }^{2}$, M. De Curtis ${ }^{3}$, G. Salvia ${ }^{4}$, L. Lega ${ }^{5}$, F. Messina ${ }^{6}$, R. Berni Canani ${ }^{7}$ \\ ${ }^{1}$ Department of Women Health and Perinatal Medicine, University "La Sapienza", Roma, ${ }^{2}$ AORN Monaldi, \\ Naples, ${ }^{3}$ University "La Sapienza", Roma, ${ }^{4}$ Hospital FBF Buon Consiglio, Naples, ${ }^{5}$ Hospital Meyer, \\ Florence, ${ }^{6}$ Hospital 'V. Betania', ${ }^{7}$ University Federico II, Naples, Italy
}

Background and aims: Gastric acidity is a major non-immune defense mechanism against infections. In adults and children treated with gastric acidity inhibitors (GAI) there is evidence of an increased risk of intestinal and extraintestinal infections. These drugs are being increasingly used in the neonatal period. To investigate the possible association between GAI and infections or necrotizing enterocolitis (NEC) in verylow-birthweight (VLBW) newborns.

Methods: VLBW consecutively observed in Neonatal Intensive Care Units for at least 8 weeks were evaluated and the effect of GAI exposure on the rate of infections and NEC was analysed.

Results: As many as 274 VLBW infants were evaluated 183 not exposed to GAI and 91 received ranitidine. The main clinical and demographic characteristics were not different between the two groups. The rate of infections was significantly higher in neonates receiving ranitidine (34/91) compared to subjects not receiving GAI $(18 / 183, \mathrm{p}<0.001)$. The number of infants presenting sepsis $(23 / 91 \mathrm{vs} 16 / 183)$, pneumonia (4/91 vs $1 / 183)$, urinary tract infections $(7 / 91$ vs $1 / 183)$ was significantly higher in the newborns receiving ranitidine than in controls $(\mathrm{p}<0.05$ ). The risk of infections (OR 5.47, 95\% CI 2.9-10.4) and NEC (OR 6.58, $95 \%$ CI 1.7-25.0) were significantly increased in neonates exposed to ranitidine.

Conclusions: Ranitidine therapy is associated with an increased risk of infections and NEC in VLBW infants. Neonatologists need to consider that the benefits of treatment outweigh the potential risk. 